CIE N CIA
ergO-SUM

\title{
Afrontamiento y síntomas de estrés postraumático en niños mexicanos después del sismo de 2017
}

\footnotetext{
González Arratia López Fuentes, Norma I vonne; Torres Muñoz, Martha Adelina; Aguilar Montes de Oca, Yessica Paola; González Arratia Visoso, Felipe; González Escobar, Sergio; Ruíz Martínez, Ana Olivia Afrontamiento y síntomas de estrés postraumático en niños mexicanos después del sismo de 2017

CIENCIA ergo-sum, vol. 26, núm. 3, noviembre 2019-febrero 2020| e60 Universidad Autónoma del Estado de México, México
}

Esta obra está bajo una Licencia Creative Commons Atribución-NoComercial-SinDerivar 4.0 Internacional.

González Arratia López Fuentes, N. I., Torres Muñoz, M. A., Aguilar Montes de Oca, Y. P., González Arratia Visoso, F., González Escobar, S. y Ruíz Martínez, A. O. (2019). Afrontamientoy síntomas de estrés postraumático en niños mexicanos después del sismo de 2017. CIE NCIA ergo-sum, 26(3). https://doi.org/10.30878/ces.v26n3a3 


\title{
Afrontamiento y síntomas de estrés postraumático en niños mexicanos después del sismo de 2017
}

Coping and Symptoms of Pos-traumatic Stress in Mexican Children after the Earthquake of 2017

\author{
Norma Ivonne González Arratia López Fuentes \\ Universidad Autónoma del Estado de México, México \\ nigalf@yahoo.com.mx \\ Martha Adelina Torres Muñoz \\ Universidad Autónoma del Estado de México, México \\ nina_156ch@hotmail.com \\ Yessica Paola Aguilar Montes de Oca \\ Universidad Autónoma del Estado de México, México \\ amarem_ypam@hotmail.com \\ Felipe González Arratia Visoso \\ Universidad Autónoma del Estado de México, México \\ felipegonzalezav@gmail.com \\ Sergio González Escobar \\ Universidad Autónoma del Estado de México, México \\ sergioglz4@hotmail.com \\ Ana Olivia Ruiz Martinez \\ Universidad Autónoma del Estado de México, México \\ ana_olivi@yahoo.com.mx
}

Recepción: 06 de abril de 2018

Aprobación: 20 de septiembre de 2018

\section{RESUMEN}

Se analiza la relación entre el afrontamiento frente a riesgos extremos y los síntomas de estrés postraumático en niños que experimentaron el terremoto de México de 2017. Al mes del evento, se evaluaron 178 niños(as) entre 11 y 15 años de edad $(M=12.90, D E=$ 1.05) de Malinalco, municipio al sur del Estado de México, con la escala de afrontamiento frente a riesgos extremos y la escala infantil de síntomas del trastorno de estrés postraumático (TEPT). Los resultados mostraron puntajes altos en TEPT en las niñas. Asimismo, el afrontamiento tanto activo como pasivo aumenta débilmente la presencia de síntomas de TEPT. Se sugiere analizar la capacidad resiliente ante este tipo de desastres naturales, así como darle un seguimiento.

Palabras clave: afrontamiento, estrés postraumático, niños, sismo, resiliencia.

\begin{abstract}
The relationship between coping with extreme risks and symptoms of post-traumatic stress in children who experienced the 2017 earthquake in Mexico was examined. One month after the event, 178 children between 11 and 15 years old $(M=12.90, S D=1.05)$ from the municipality of Malinalco, were evaluated with Coping Scales Facing Extreme Risks and the Childhood Scale of Symptoms of Post-traumatic Stress Disorder (PTSD) in the South of the State of Mexico. The results showed high scores in PTSD, especially in girls, and both, the active and the passive coping rise weakly the presence of symptoms of PTSD. It is suggested analyzing the resilient ability with respect to the exposure to this type of natural disasters, and carry out follow-up studies.
\end{abstract}

KEYWORDS: coping, post-traumatic stress disorder, children, earthquake, resilience. 


\section{INTRODUCCIÓN}

El estudio sobre los desastres naturales que contemple variables psicológicas no es nuevo, ya que la investigación al respecto ha sido ampliamente documentada, en especial en muestras de adultos y niños que han sido víctimas de huracanes (Osofsky et al., 2009). La literatura indica que en su mayoría los estudios se han enfocado en la población adulta; sin embargo, los realizados en muestras infantiles que han experimentado un desastre natural, como los terremotos, son escasos.

El sismo del pasado 19 de septiembre de 2017 en México tuvo una magnitud de 7.1 grados ML, con profundidad de $51 \mathrm{~km}$, y se registró a las 13:14 h, tiempo del centro, según lo reportó el Servicio Sismológico Nacional (SSN, 2017); su intensidad corresponde a un movimiento severo con altas probabilidades de causar daño que a su vez originó múltiples réplicas. Entre los estados que presentaron mayores daños se encuentra el Estado de México. Malinalco fue el municipio que resultó más afectado (Diario Basta, 2017), razón por la que se eligió esta zona para llevar a cabo la investigación de este artículo.

Cuando se vive un evento catastrófico de esta naturaleza, los principales sentimientos que evidencia la población son el miedo, la incertidumbre y el estrés. En este sentido, investigaciones previas indican que la existencia de amenazas potenciales como los terremotos tiene impacto sobre la salud mental de los individuos (Pynoos et al., 2007).

Se ha observado que ante la exposición de riesgos la mayoría de los niños y adolescentes enfrenta con éxito y demuestra habilidades de adaptación después de un trauma (Benigth y Bandura, 2004), mientras que para otros no es tan fácil, ya que incluso puede convertirse de una experiencia considerada como amenaza a una experiencia traumatizante. Si bien durante el periodo escolar y la preadolescencia los individuos están capacitados para juzgar el nivel de gravedad de una amenaza (Rodríguez et al., 2015), es indispensable analizar cuál es el impacto que causan estos eventos cuando es vivenciado por los niños, esto es, comprender cuáles son las estrategias para afrontar los desastres y la relación existente con la presencia de síntomas de estrés postraumático.

Lo anterior se debe a que en cualquier momento hay que hacer frente a catástrofes naturales y peligros potenciales que pongan en riesgo la vida de los individuos y generen un importante estrés psicológico, por lo que la configuración del entorno se evalúa como ambigua generalmente (Lazarus y Folkman, 1986) y ante ello es difícil tener confianza respecto a la forma en cómo hay que actuar. De esta manera, el hecho de contar con información sobre la relación entre estos factores es indispensable para recuperar la salud y bienestar ante un eventual desastre (Rodríguez et al., 2015).

El afrontamiento, desde la postura cognitivo conductual de Lazarus y Folkman (1986: 164), se define como: "aquellos esfuerzos cognitivos y conductuales constantemente cambiantes que se desarrollan para manejar las demandas específicas externas y/o internas que son evaluadas como excedentes o desbordantes de los recursos del individuo".

Generalmente se distinguen dos tipos de estrategias ante una situación demandante: a) las estrategias de solución de problemas y b) las estrategias de regulación de emociones, que Moos y Billings (1986) proponen como a) afrontamiento activo y $b$ ) afrontamiento pasivo. El primero está dirigido a comportamientos de acción directa sobre el problema, búsqueda de información, estrategias de anticipación a un desastre, control de sí mismo y de las circunstancias y soporte social, mientras que el afrontamiento pasivo se refiere a la negación o evitación del problema, comportamientos de rechazo, retraimiento y aceptación pasiva. En el proceso para enfrentar las dificultades y resultado final pueden intervenir múltiples factores tanto internos (variables de personalidad) como externos (apoyo social, recursos materiales), los cuales pueden modular el impacto de un estresor (Vázquez Valverde et al., 2003). También se ha referido que la elección de alguna estrategia según Baggio y Rouquette (2006) puede ser por el sentimiento de implicación de las personas frente a la amenaza. 
Aguilar-Mendoza (2016) reportó que si bien se presentan niveles altos de afrontamiento activo en familias ante riesgo de desastres naturales, también existe un número mayor de familias que tienden al afrontamiento de tipo pasivo. Del mismo modo, Villagrán et al. (2014) reportaron que las personas que sufrieron el terremoto de Chile en 2010 manifiestan un estilo de afrontamiento activo. Otros estudios en muestras latinoamericanas han puesto en evidencia que los individuos que tienden a utilizar estrategias de afrontamiento pasivas muestran mayor incapacidad para resolver problemas y también menor bienestar (Salotti, 2006).

La investigación al respecto indica que la población mexicana frente al terremoto ocurrido en 1985 presentó la tendencia de "no hacer nada frente al riesgo sísmico, aceptando las pérdidas en caso de que un evento de este tipo se repitiera" (López-Vázquez y Marván, 2004: 217). En este sentido, según Shalev (2005: 119), "las personas traumatizadas están atrapadas en un círculo vicioso realizando esfuerzos inapropiados por hacer frente a un estresor y pierden la capacidad de desprenderse". Entre las explicaciones a esto se encuentra que las estrategias de afrontamiento adaptativas se relacionan con un entorno con bajo nivel de incertidumbre, mientras que las situaciones inesperadas o extremas generan respuestas desadaptativas (Lazarus y Folkman, 1986; Salgado y Leria, 2018).

Por otro lado, las consecuencias psicológicas después de los desastres son diversas; por ejemplo, se ha documentado que no afectan de igual forma a todas las personas y en algunos casos puede dejar en ellas una serie de síntomas del síndrome de estrés postraumático (TEPT), el cual es muy estudiado (Caro y Cova, 2012). A pesar de que pueden encontrarse diversas conceptualizaciones, en términos generales se entiende como "una respuesta sintomatológica que una persona desarrolla después de haber estado expuesta a un suceso altamente estresante, debido a que ha amenazado su integridad física o su vida, o la de otras personas" (Medina-Mora et al., 2005: 8).

De acuerdo con el DSM-5 (American Psychiatric Association, 2014), el TEPT se caracteriza por una serie de síntomas que incluyen a) la reexperimentación del evento traumático en forma de pesadillas o imágenes constantes e involuntarias, $b$ ) conductas de evitación asociadas a la situación o hecho traumático y $c$ ) respuestas de hiperactivación (e.g. dificultades para concentrarse). "La alteración causa malestar clínicamente significativo o deterioro en lo social, laboral u otras áreas importantes de funcionamiento, y la alteración no se puede atribuir a los efectos fisiológicos de una sustancia o a otra afección médica” (American Psychiatric Association, 2014: 161 164). Además, como un criterio importante, es que los síntomas duren más de un mes y se produzca un malestar clínicamente significativo.

En el mundo se estima que al menos $60 \%$ de las personas ha experimentado uno o más acontecimientos traumáticos en algún momento de su vida (Kessler et al., 2014). Aunque también se han documentado variaciones respecto a la prevalencia, en el caso de los niños se ha reportado 8\% de TEPT clínicamente significativo, e incluso se ha presentado ocho meses después de la vivencia de un desastre natural como lo fue el huracán Ike (Lai et al., 2013).

En México Tapia et al. (1987) reportaron que 32\% de personas damnificadas por el terremoto en México de 1985 presentaba TEPT con síntomas que incluían angustia generalizada, agitación, temblor, dificultad para concentrarse, trastornos del sueño, los cuales incluso continuaron hasta un mes después del terremoto. Por sexo, Medina-Mora et al. (2005) han reportado una prevalencia de trastorno por TEPT en el caso de adultos de $2.3 \%$ en las mujeres y $0.49 \%$ en los hombres.

Las prevalencias más elevadas fueron las asociadas a la persecución o acoso, la violación, el secuestro, el abuso sexual y a ser víctima de violencia por parte de los padres. También, se ha señalado la hipótesis de que las características preexistentes de la personalidad del individuo pueden intervenir en la génesis de las reacciones psicológicas posteriores a un desastre. Un ejemplo de ello es la investigación de Neuner et al. (2006), quienes indican la existencia de factores previos al desastre como edad, raza y género, los cuales se asocian con mayor probabilidad al estrés postraumático.

Específicamente en cuanto al sexo, se ha observado discrepancia en los hallazgos, ya que Tapia et al. (1987) señalan que las mujeres presentaron una frecuencia de reacciones psicológicas tres veces mayor a la de los hombres sin importar la edad ni la escolaridad. Al respecto, el estudio de Giannopoulo et al. (2006) reporta que las niñas 
presentan más síntomas de estrés postraumático después del terremoto de Atenas en 1999 en comparación con los niños. Sin embargo, también se ha reportado que en sucesos como los desastres naturales no se observan diferencias entre los sexos (Medina-Mora et al., 2005).

Entre las explicaciones de estas diferencias se ha observado que las mujeres tienen mayor probabilidad de desarrollar TEPT debido a que denotan una mayor percepción de amenaza y pérdida de control ante el trauma, mayor reacción emocional, menor apoyo social y tienden a utilizar estilos de afrontamiento de evitación (Olff et al., 2007).

Por otro lado, en estudios previos se ha demostrado que existe una relación moderada entre el estilo de afrontamiento y el estrés postraumático en estudiantes que vivieron el terremoto y tsunami de 2010 en Chile (Rincón et al., 2014). Otra evidencia es la investigación de Caro et al. (2012) en donde indican que a mayores niveles de estrés postraumático los individuos exhiben menores niveles de afrontamiento represivo.

Con base en lo anterior, resulta relevante estudiar los efectos del evento acontecido el pasado 19 de septiembre de 2017. De este modo, los propósitos de este artículo son a) analizar la relación que existe entre el afrontamiento frente a riesgos extremos y el estrés postraumático en niños y niñas que se vieron sometidos a este riesgo, $b$ ) describir la sintomatología postraumática que presentan y $c$ ) describir las diferencias que presentan estas variables entre niños y niñas.

El interés por este estudio radica, como lo refieren Kellens et al. (2013) y Navarro et al. (2016: 343), en el sentido de que es "necesario generar investigación sobre las amenazas ambientales actuales y cómo pueden tener un efecto sobre los comportamientos de protección, formas de adaptación individual y colectiva” con el fin de implementar estrategias de atención en casos de desastres naturales.

\section{Método}

\section{1. Participantes}

Se trata de un estudio no experimental transversal con una muestra de tipo intencional en el que participaron 181 niños (de los cuales se reportan tres datos perdidos, por lo que se trabajó con un total de 178) que vivenciaron el terremoto del 19 de septiembre de 2017. Todos son habitantes de la comunidad de San Simón el Alto, perteneciente al municipio de Malinalco, al sur del Estado de México, donde las casas de la zona sufrieron importantes afectaciones. El rango de edad fue de 11-15 años $(M=12.90, D E=1.05)$, 85 niños (47\%) y 93 niñas $(53 \%)$. Todos los participantes pertenecen a una secundaria pública (primer grado 63, segundo grado 60 y tercer grado 55), fueron evaluados un mes después del suceso y reportaron estar en la escuela durante el sismo.

\section{2. Instrumentos}

Se aplicó la Escala de Afrontamiento Frente a Riesgos Extremos que mide las estrategias de afrontamiento de los sujetos que viven en situaciones de riesgo natural o industrial (López-Vázquez y Marván, 2004). Consta de 26 ítems y los autores reportan índice de consistencia interna alfa de Cronbach de 0.81 y $31.65 \%$ de la varianza total explicada con dos factores: $a$ ) afrontamiento activo $(\alpha=0.79$, 14 ítems y $17.71 \%$ de la varianza) y $b$ ) afrontamiento pasivo ( $\alpha=0.76,12$ ítems y $13.93 \%$ de la varianza). En este estudio se obtuvo un alfa de Cronbach de 0.79 para la escala total.

Asimismo, se aplicó la Escala de Síntomas del TEPT para niños (CPSS): instrumento de autoinforme desarrollado por Foa et al. (2001) para evaluar la presencia de síntomas del TEPT en población infanto-juvenil con una historia conocida de trauma. Está basada en los criterios diagnósticos del DSM-IV y consta de 17 síntomas para el diagnóstico; en ella el sujeto debe puntuar la frecuencia de cada uno durante las últimas dos semanas en una escala de cinco puntos (va de nunca a nueve veces y más). La frecuencia en la que se presenta la situación considera un marco de referencia temporal de una semana previa y tampoco se incluyó la escala complementaria. En este 
estudio se aplicó la versión validada por Rincón et al. (2014) en niños (as) y adolescentes chilenos de 8 a 18 años de edad expuestos a desastres naturales por considerarse que es una versión más cercana al español hablado en México. Cuenta con tres subescalas que abarcan los síntomas reexperimentación ( $\alpha=0.75,5$ ítems), evitación ( $\alpha=0.66,7$ ítems $)$ y activación $(\alpha=0.70,5$ ítems). Los puntajes mayores a 24 o más puntos permiten obtener una sensibilidad de $82 \%$ y especificidad de $88 \%$. En este artículo se obtuvo una fiabilidad alfa de Cronbach de 0.89.

\subsection{Procedimiento}

Se solicitó el consentimiento informado por parte de los padres, así como la autorización de la institución. Las aplicaciones de ambas escalas se llevaron a cabo un mes después de ocurrido el evento dentro de las respectivas aulas y de manera colectiva en un tiempo aproximado de $45 \mathrm{~m}$, además se aclararon las dudas que surgieron en esos instantes.

\subsection{Análisis de datos}

Se procedió a hacer análisis descriptivos de las escalas (media y desviación estándar), así como de fiabilidad (alfa de Cronbach). Posteriormente, se realizó un análisis de diferencias entre niños y niñas para cada variable, el cual utilizó la prueba $t$ de Student para muestras independientes. A través de coeficientes de correlación de Spearman se examinó la relación entre afrontamiento y TEPT, de la cual se consideró un nivel de confianza de 95\%. Los análisis se llevaron a cabo con el programa SPSS-23.

\section{Resultados}

En el cuadro 1 se presentan los datos descriptivos de la muestra total. Los puntajes más altos se observan en el caso de la subescala de afrontamiento activo, mientras que en afrontamiento pasivo son menores. En el caso de las subescalas de TEPT se aprecia que es menor la presencia de síntomas de activación y en el caso de la subescala de evitación resultó ser la más alta. Del puntaje global de esta escala, y de acuerdo con el punto de corte según el criterio de Rincón et al. (2014) con un valor de 24 o más puntos, se obtuvo que el promedio de la muestra total se encuentra por arriba de este criterio $(M=25.29)$. Respecto a la consistencia interna de ambas escalas, resultaron ser satisfactorias, excepto en la dimensión de afrontamiento pasivo que es baja, según el criterio de Oviedo y Campo-Arias (2005).

\section{CUADRO 1}

Datos descriptivos de las escalas de Afrontamiento y TEPT

\begin{tabular}{llrrrrr}
\hline & & Mín.-Máx. & $\boldsymbol{M}$ & $\boldsymbol{D E}$ & Alfa de Cronbach & Núm. de ítems \\
\hline \multirow{2}{*}{ Afrontamiento } & Activo & $18-64$ & 40.77 & 9.08 & 0.802 & 14 \\
& Pasivo & $20-47$ & 32.89 & 5.29 & 0.528 & 12 \\
\hline \multirow{2}{*}{$\begin{array}{l}\text { Síntomas de estrés } \\
\text { postraumático }\end{array}$} & Evexperimentación & $0-20$ & 8.54 & 4.92 & 0.822 & 5 \\
& Activación & $0-24$ & 9.37 & 5.71 & 0.719 & 7 \\
& Puntaje total TEPT & $0-20$ & 7.31 & 4.87 & 0.788 & 5 \\
\hline
\end{tabular}

Fuente: elaboración propia. 
Posteriormente, se realizó una comparación por sexo por medio de la prueba $t$ de Student. Los resultados mostraron que en las subescalas de afrontamiento no existen diferencias significativas entre niños y niñas, mientras que en el caso de síntomas de estrés postraumático las niñas reportan puntajes promedio más altos en reexperimentación, evitación y activación (cuadro 2).

\section{CUADRO 2}

Diferencias según sexo para estrategias de afrontamiento y TEPT

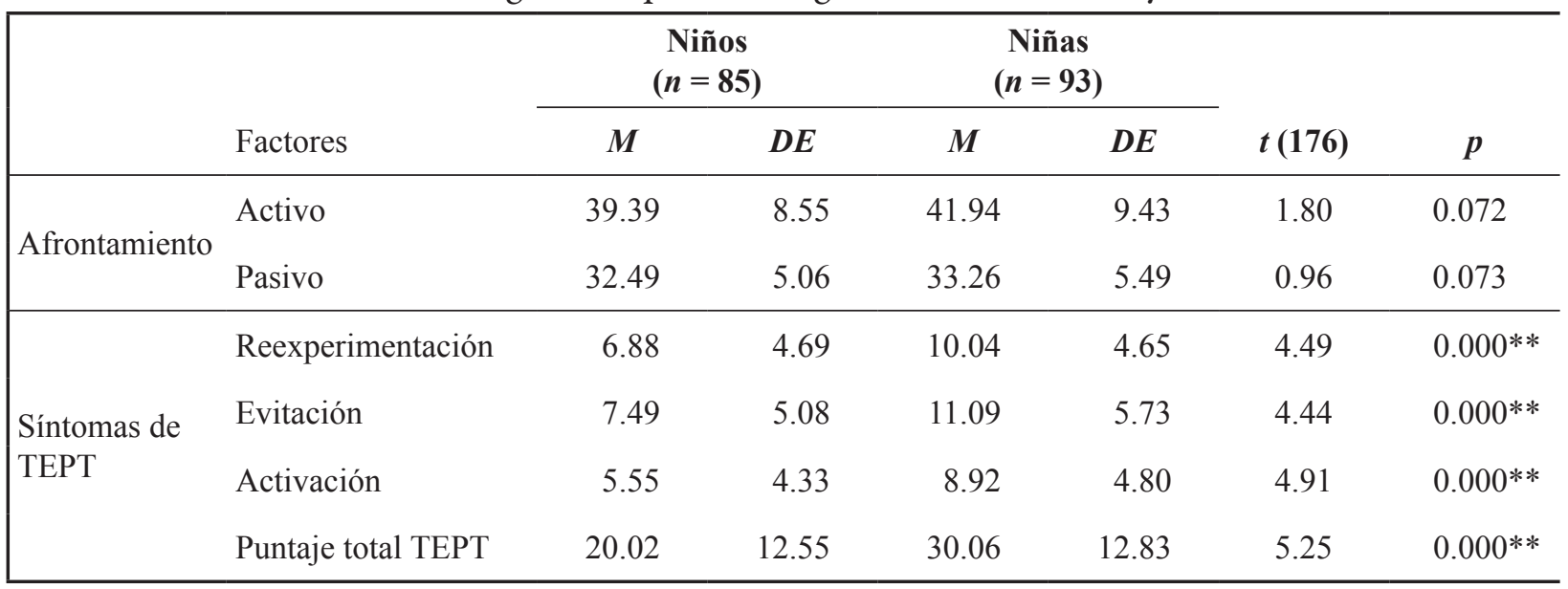

Fuente: elaboración propia.

Nota: ${ }^{* *} p<0.01$

Para diferenciar a los individuos con y sin síntomas de TEPT se consideró como referencia el criterio de Rincón et al. (2014); en este caso, se encontró que 52.8\% de la muestra presenta algún tipo de síntoma de estrés, de ella 30 son niños y 64 niñas. Así, los participantes que puntuaron menor a 24 constituyeron el grupo 1 (sin síntomas de TEPT) y aquellos con un puntaje superior a 24 conforman el grupo 2 (con síntomas de TEPT). Después, se llevó a cabo un análisis entre ambos con la finalidad de observar las posibles diferencias según las estrategias de afrontamiento por medio de la prueba $t$ de Student. En el cuadro 3 se observan las medias que indican que los niños/as clasificados/as en el grupo con síntomas de TEPT presentan puntajes más altos en estrategias de afrontamiento tanto activo como pasivo.

\section{CUADRO 3}

Diferencias por grupo con y sin síntomas de EPT según estilos de afrontamiento

\begin{tabular}{|c|c|c|c|c|c|c|c|}
\hline & \multirow[b]{2}{*}{ Factores } & \multicolumn{2}{|c|}{$\begin{array}{c}\text { Sin síntomas } \\
\text { TEPT } \\
(n=84)\end{array}$} & \multicolumn{2}{|c|}{$\begin{array}{c}\text { Con síntomas } \\
\text { TEPT } \\
(n=94)\end{array}$} & \multirow[b]{2}{*}{$t(176)$} & \multirow[b]{2}{*}{$p$} \\
\hline & & Media & $D E$ & Media & $D E$ & & \\
\hline \multirow{2}{*}{ Afrontamient } & Activo & 39.11 & 9.51 & 42.30 & 8.48 & -2.34 & 0.020 \\
\hline & Pasivo & 31.72 & 5.81 & 33.95 & 4.95 & 2.79 & 0.006 \\
\hline
\end{tabular}

Fuente: elaboración propia.

Del análisis de correlación, los datos muestran una relación positiva significativa entre la subescala de afrontamiento activo y los síntomas de reexperimentación, activación y el total de TEPT; la relación con la subescala de evitación no resulta significativa. Respecto a la subescala de afrontamiento pasivo, hay relación significativa con los tres síntomas y el total de la escala de TEPT. Todas las correlaciones se evidencian débiles (cuadro 4). 


\section{CUADRO 4}

Correlaciones entre la escala de Afrontamiento y TEPT

\begin{tabular}{lcc}
\hline & Afrontamiento activo & Afrontamiento pasivo \\
\hline 1. Reexperimentación & $0.25^{* *}$ & $0.25^{* *}$ \\
2. Evitación & - & $0.21^{* *}$ \\
3. Activación & $0.23^{* *}$ & $0.24^{* *}$ \\
4. Puntaje total síntomas estrés & $0.22^{* *}$ & $0.26^{* *}$ \\
\hline
\end{tabular}

Fuente: elaboración propia.

Nota: ${ }^{* *} p<0.01$

\section{Discusión}

A partir de los resultados encontrados se observó que los participantes tienden a utilizar en mayor medida un estilo de afrontamiento activo, lo que indica la presencia de comportamientos de acción directa ante un evento como el pasado terremoto del 19 de septiembre de 2017. Esto coincide con Aguayo et al. (2016) y López-Vázquez y Marván (2015), ya que cuando se trata de riesgos naturales un alto nivel de estrés psicológico predice el uso de estrategias activas. Del mismo modo, se ha reportado que "los individuos expuestos a riesgos naturales (como inundaciones) se enfocan más frecuentemente en estrategias de afrontamiento activo” (Navarro et al., 2016: 339).

En cuanto a TEPT los datos están ligeramente por arriba del punto corte. Este resultado indica que los niños manifiestan reacciones en los tres síntomas de estrés postraumático, especialmente en el ítem 3 (“¿Has tenido la sensación como si lo que te pasó estuviera ocurriendo de nuevo, como si estuvieras ahí otra vez?”). Dicho ítem pertenece a la dimensión reexperimentación, en donde $25.3 \%$ indica que en las últimas dos semanas se ha presentado nueve veces y más. Si bien estos datos sugieren la presencia de estos síntomas, es indispensable precisar este diagnóstico no sólo con la aplicación del instrumento, sino también incluir otras pruebas psicológicas, así como entrevistas clínicas con el fin de verificar la presencia del trastorno y emprender las acciones correspondientes.

Respecto al sexo no hubo diferencias en lo concerniente al estilo de afrontamiento, lo que es opuesto a lo planteado inicialmente. Si bien es cierto que estos resultados son contrarios a lo que gran parte de la literatura menciona respecto a las diferencias entre hombres y mujeres, los hallazgos de este trabajo coinciden con Frydenberg y Lewis (1991) y Compas et al. (2001), quienes indican que no existen diferencias en los hombres y mujeres en la forma como enfrentan el estrés. Una posible explicación es que estos resultados pueden deberse a un reflejo de la situación actual y a los niveles de igualdad alcanzados entre hombres y mujeres (Martín et al., 2011). Sin embargo, es necesario continuar verificando este patrón de afrontamiento que tienen los hombres y las mujeres en el caso de enfrentarse a situaciones extraordinarias.

En cuanto a la sintomatología de TEPT los datos indican que es superior en las mujeres y esto es visible en investigaciones como la de López-García y López-Soler (2014) y Tolin y Foa (2006), quienes reportan que estas diferencias se mantienen en la infancia, adolescencia y la edad adulta, por lo que, al igual que López-García et al. (2014: 232), se constata que en especial "las niñas deberían considerarse un grupo especial de riesgo".

En las diferencias por grupo se observa que los participantes con síntomas de TEPT suelen usar estrategias de afrontamiento tanto activas como pasivas. Si bien el uso de estrategias activas se considera más adecuado y funcional ante estos acontecimientos, también lo es el hecho de que exista la tendencia por las estrategias pasivas. Es probable que lo anterior se deba a varias razones: una de ellas es el hecho de generar cierta sensación de desahogo y alivio ante la circunstancia de estrés, otra es la etapa de desarrollo de los participantes, ya que se ha visto que "el afrontamiento se hace más efectivo con la edad" (Lazarus y Folkman, 1986: 194). También puede deberse al impacto de la situación, pues "cuanto mayor es la amenaza, más se tiende a usar estrategias 
dirigidas a la emoción" (Lazarus et al., 1986: 190), así como la forma en que es evaluada la situación, ya que, si es evaluada como una amenaza extrema, interfiere con el modo de afrontamiento dirigido al problema. Lo cierto es que en estos casos se debe considerar que el afrontamiento es multicausal. Por esta razón, los resultados obtenidos llevan a la reflexión de que posiblemente variables como la autoestima cambian el significado de los sucesos ambientales (Verduzco et al., 2004), de tal forma que se requiere continuar con la investigación y así contar con mayor evidencia al respecto.

En el caso de la relación entre las variables de interés, este estudio constata la asociación entre estilos de afrontamiento y TEPT, que es coherente con el estudio de Rincón et al. (2014). Aunque estos datos indican que afrontar de manera activa un acontecimiento extremo se asocia con mayores síntomas de reexperimentación y activación, también se encontró que hacer uso de estrategias de afrontamiento pasivo genera síntomas de TEPT. Esto podría explicarse en el sentido de que los participantes tienden a usar ambas estrategias de afrontamiento. Al respecto, en otros estudios hechos en México por López-Vázquez et al. (2015) en individuos que no fueron evacuados de zonas afectadas por fenómenos hidrometeorológicos reportaron que la percepción de riesgo, el estrés psicológico y TEPT correlacionan con el afrontamiento activo y con afrontamiento pasivo de manera moderada y significativa, lo que sugiere más el uso de afrontamiento flexible. Así, estos resultados coinciden con el modelo de afrontamiento flexible propuesto por Chen et al. (2012) respecto a la capacidad de utilizar estrategias de afrontamiento tanto activas como pasivas que hacen posible una mejor salud, es decir, según la situación y la conveniencia, la persona puede usar una u otra estrategia para adaptarse y enfrentar de un mejor modo la situación. También, se hace el señalamiento que las estrategias de afrontamiento tanto activas como pasivas tienen que ver con aspectos culturales (López-Vázquez y Marván, 2004), por lo que es posible que en el caso de la muestra bajo estudio los individuos tienden a usar estrategias tanto centradas en el problema como en las emociones para hacer frente a la situación a la que estuvieron expuestos. Sin embargo, esto hace que sea necesario evaluar la percepción de riesgo que poseen los participantes, puesto que no todas las personas se ven afectadas de la misma forma por los estresores psicosociales ni tampoco responden del mismo modo ante las situaciones de estrés (Lazarus et al., 1986; Vázquez Valverde et al., 2003).

Todo esto lleva a concluir que los participantes mostraron sintomatología de TEPT. Las mujeres en su caso son quienes manifiestan puntuaciones significativamente más altas. Los análisis también mostraron que no hay diferencias en las estrategias de afrontamiento (pasivo y activo) entre hombres y mujeres y se comprobó la relación entre TEPT y estrategias de afrontamiento ante los desastres. Por esto, el hecho de vivir la experiencia de un terremoto es posible que incremente el TEPT. Sin embargo, por tratarse de un estudio de tipo correlacional, sólo se hace referencia a la asociación entre estas variables, por lo que no es posible comprobar una posible causalidad. $\mathrm{Al}$ momento de analizar las estrategias de afrontamiento se observa que no hay presencia de un estilo único, sino que se da tendencia a presentar ambas estrategias, lo cual hace suponer que el uso de una u otra dependen de las demandas del entorno.

Desde la perspectiva de los autores de este artículo, se reconoce que el hecho de vivir este tipo de experiencias requiere de la identificación del posible daño emocional o severidad del trauma en los niños y adolescentes. Aunado a esto, es indispensable establecer estrategias que permitan la salud mental de los individuos y logren un mejor ajuste y adaptación, además de incluir el estudio sobre la capacidad resiliente de los participantes en caso de desastre; sin duda, esto ayudaría a explicar aún más el comportamiento ante la vivencia de un terremoto e incidir en el bienestar de las personas al tiempo de generar una cultura de protección.

Es importante destacar que este estudio presenta varias limitaciones: a) por una parte el diseño de investigación, ya que se trata de un estudio trasversal y sería necesario dar un seguimiento a los participantes con un estudio de tipo longitudinal y así constatar la presencia de síntomas de estrés postraumático; para conseguirlo se requiere de una segunda medición a los mismos sujetos y contar con indicadores de prevalencia de TEPT. Al mismo tiempo, continuar con el análisis de las estrategias de afrontamiento de los niños después del segundo sismo, como el del pasado febrero del 2018, para conocer el impacto en su comportamiento. 
b) Por otra, esta investigación trata de un estudio a nivel correlacional, por lo que aún es necesario continuar con el análisis de la posible interacción de las variables que den una mayor explicación de las distintas estrategias de afrontamiento y establecer el diagnóstico de TEPT; aplica lo mismo para el tipo de muestreo utilizado que resulta en hallazgos no generalizables y sólo sean para los participantes bajo estudio.

Con la información que se dispone, surge ahora la necesidad de incluir en una investigación futura el análisis de otros síntomas como el estrés, la ansiedad y la depresión, las cuales se encuentran vinculadas con las experiencias de desastres naturales, así como el apoyo social con el que cuentan las personas en situaciones de emergencia y desastres. También es posible que estos resultados se deban a que la experiencia ante estos sucesos afecta la percepción que se tiene del peligro y por consiguiente en la forma como se afronta, de ahí que sea indispensable incorporar la medición acerca de cómo se evalúa el riesgo y en qué momento de la vida para así entender su función en el ajuste psicológico de los niños cuando han sido expuestos a este tipo de desastres naturales.

Finalmente, coincidimos con Bernaldes et al. (2017) cuando refieren que es mejor estar preparados frente a un eventual sismo, tanto en lo individual como en lo colectivo, para enfrentar los desastres naturales. Por eso, es fundamental que se generen programas de prevención en diferentes grupos de edad para fortalecer a distintos grupos tal y como lo indica Aguilar-Mendoza (2016): unos para actuar y otros para cuidar y proteger, como es el caso de los niños, y qué mejor si se hace desde la institución educativa.

\section{AnÁlisis Prospectivo}

Los desastres naturales conllevan pérdidas humanas o económicas. Esta situación puede generar la necesidad de que intervengan diferentes áreas. Desde la psicología, por ejemplo, implica el reconocimiento de que posiblemente la emoción experimentada por las personas durante y después del terremoto fue abrumadora y angustiante trayendo como consecuencia efectos traumáticos e importantes efectos sobre la salud mental de los individuos (Bambarén, 2011). Por estos motivos, es indispensable dirigir nuestra mirada hacia un trabajo que permita la reintegración homeostática desde la resiliencia a fin de recuperarse y tener un funcionamiento óptimo, así como también analizar si la vivencia, como la de este estudio, genera en ellos un cambio positivo que los dirija a un crecimiento postraumático, el cual parta de la consideración de que los contratiempos pueden ser una preparación psicológica ante la adversidad. Con base en esto, la pregunta sería ahora: ¿Qué es lo que han aprendido de esta experiencia? Y que esto permita la identificación de áreas de oportunidad propias que no se habían descubierto sino después de la situación.

Por otro lado, desde la política de protección civil estos hallazgos son útiles, ya que el conocimiento de los efectos emocionales que sufrieron los individuos y sus modos de enfrentarse a situaciones extremas pueden dar la pauta de cómo incrementar "la cultura de prevención ante los riesgos a la población, la cual debe considerar las particularidades socioculturales de cada región" (Fernández y Toscana, 2018: 7).

Si bien es cierto que un sismo no se puede predecir, está latente la probabilidad de que suceda de nuevo debido a que la comunidad se encuentra en una zona sísmica, de ahí su vulnerabilidad. De esta manera, se requiere la preparación y empleo de estrategias de prevención en la comunidad, así como continuar incentivando el desarrollo de simulacros ante los sismos para minimizar en la medida de lo posible el daño a la población expuesta.

\section{Agradecimientos}

Agradecemos a las autoridades de la institución por el apoyo recibido para llevar a cabo esta investigación y en especial a todos los participantes.

Esta investigación forma parte del Proyecto de Investigación titulado "Mediadores y Moderadores de la Resiliencia en niños y adolescentes". y registrado en la UAEM con clave 4222/2016SF, así como del proyecto con clave 4645/2019SF. 


\section{ReFERENCIAS}

Aguayo, M. A., López, V. E., Lorenzo, R. A., Ávila, G. M. E., Vera, J. J. A. y Hindrichs, I. (2016). Estrategias de afrontamiento ante emergencias y desastres. Cuadernos de crisis y emergencias, 15(2) Disponible en http://www.cuadernosdecrisis.com/docs/2016/numero15vol2_2016.pdf. Consultado el 10 de marzo de 2018.

Aguilar-Mendoza, A.S. (2016). El afrontamiento y la capacidad de resiliencia de las personas ante los riesgos por desastres naturales. Revista Entorno, 62, 34-46.

American Psychiatric Association (APA). (2014). Diagnostic and Statistical Manual of Mental Disorders (DSM5) (5th ed.) Arlington: APA.

Bados, A. (2015). Trastorno por estréspostraumático. Facultad de Psicología. Disponible en http://diposit.ub.edu/ dspace/bitstream/2445/65623/1/TEPT.pdf. Consultado el 26 de febrero 2018.

Baggio, S., \& Rouquette, M. L. (2006). La représentation sociale de l'inondation: influence croisée de la proximité au risque et de l'importance de l'enjeu. Bulletin de Psychologie, 59, 103-117.

Bambarén, A. C. (2011). Salud mental en desastres naturales. Revista Psicológica Herediana, 6(1-2) 20-25.

Bernaldes, M., McIntyre, A. M. y Cabieses, B. (2017). Estrategias de afrontamiento ante desastres naturales en población migrante en la región Valparaiso. Instituto de Ciencias e Innovación en Medicina. https://pdfs. semanticscholar.org/fc21/e1cb6924b50e9c98c55994d27ecd05f1d5da.pdf. 1-41.

Benight, C. C., \& Bandura, A. (2004). Social cognitive theory of posttraumatic recovery: The role of perceived self-efficacy. Behaviour Researchand Therapy, 42, 1129-1148.

Bustos, P., Rincón, P. y Aedo, J. (2009). Validación preliminar de la Escala Infantil de Síntomas del Trastorno de Estrés Postraumático (Child PTSD Symptom Scale, CPSS) en niños/as y adolescentes víctimas de violencia sexual. Psykhe, 18(2), 113-126.

Caro, M., Paz, P. y Cova Solar, F. M. (2012). Relación entre estrés postraumático, afrontamiento represivo y rumiación en estudiantes universitarios chilenos. Ciencia y Enfermería, 18(3), 121-130.

Chen, C., Kogan, A. \& Hin-man Chio, J. (2012). The effectiveness of a new, coping flexibility interventions as compared with a cognitive-behavioural intervention in managing work stress. Work \& Stress, 26(3), 272-288.

Compas, B. E., Connor-Smith, J. K., Saltzman, H., Thomsen, A., \& Wadsworth, M. E. (2001). Coping with stress during childhood and adolescence: Problems, progress potential in theory and research. Psychological Bulletin, 127, 87-127.

Diario Basta (2017). Disponible en http://www.diariobasta.com/2017/09/22/municipios-del-estado-de-mexico-afectados-sismo/. Consultado el 27 de febrero de 2018.

Fernández, P. M., y Toscana, A. A. (2018). Narrativas populares sobre desastres. El caso de Amecameca ante el riesgo volcánico del Popocatépetl. CIENCIA ergo-sum, 25(1), 1-9. Disponible en https://cienciaergosum. uaemex.mx/article/view/9281.

Foa, E., Johnson, K., Feeny, N., \& Treadwell, K. (2001). The Child PTSD Symptom Scale: A preliminary examination of its psychometric properties. Journal of Clinical Child Psychology, 30, 376-384. https://doi. org/10.1207/S15374424JCCP3003_9.

Frydenberg, E., \& Lewis, R. (1991). Adolescent coping: The different ways in which boys and girls cope. Journal of Adolescence, 14(2), 119-133. 
Giannopoulou, I., Strouthos, M., Smith, P., Dikaiakou, A., Galanopoulou, V., \& Yule, W. (2006). Post-traumatic stress reactions of children and adolescents exposed to the Athens 1999 earthquake. European Psychiatry, 21,160-166.

Kellens, W., Terpstra, T., \& De Maeyer, P. (2013). Perception and communication of flood risks: A systematic review of empirical research. Risk Analysis, 33, 24-49. https://doi.org/10.1111/j.1539-6924.2012.01844.x.

Kellens, W., Zaalberg, R., Neutens, T., Vanneuville, W., \& De Maeyer, P. (2011). An analysis of the public perception of flood risk on the Belgian coast. Risk Analysis, 31(7), 1055-1068. https://doi.org/10.1111/j.15396924.2010.01571.x

Kessler, R. C., Rose, S., Koenen, K. C., Karam, E. G., Stang, P. E., Stein, D. J. (...) Carmen Viana, M. (2014). How well can post-traumatic stress disorder be predicted from pre-trauma risk factors? An exploratory study in the WHO World Mental Health Surveys. World Psychiatry, 13(3), 265-274. https://doi. org/10.1002/wps.20150.

Lai, S. B., La Greca, A., Auslander, B. A., \& Short, M. B. (2013). Children's symptoms of posttraumatic stress and depression after a natural disaster: Comorbility and risk factors. Journal Affect Disord, 146(1), 71-78. https://doi.org/10.1016/j.jad.2012.08.041.

Lazarus, R. S., \& Folkman, S. (1986). Estrés y procesos cognitivos. Barcelona: Martínez Roca.

López Vázquez, E., y Marván, G. M. L. (2004). Validación de una escala de afrontamiento frente a riesgos extremos. Salud Pública de México, 16(3), 216-221.

López-Vázquez, E., Marván, G. M. L. y Dorantes, A. G. (2015). La evacuación por inundaciones y su impacto en la percepción de riesgo y el estrés postraumático. Psicología y Salud, 25(2), 261-271.

López-García, J. y López-Soler, C. (2014). Trastorno de estrés postraumático en escolares tras el terremoto de Lorca (España) en 2011. Gaceta Sanitaria, 28(3), 230-233.

Martín, S. A., Lucas, M. B. y Pulido, V. R. (2011). Diferencias de género en el afrontamiento en la adolescencia. BROCAR, 35, 157-166.

Medina-Mora, M. E., Borges-Guimaraes, G., Lara, C., Ramos-Lira, L., Zambrano, J. y Fleiz-Bautista, C. (2005). Prevalencia de sucesos violentos y de trastorno por estrés postraumático en la población mexicana. Salud Pública, 47(1), 8-22.

Moos, R. H., \& Billings, A. G. (1986). Conceptualizing and measuring coping resource and processes, en L. Goldberger y S. Breznitz (eds.), Handbook of stress: Theoretical and clinical aspects (pp. 212-230). New York: Free Press.

Navarro, O., Chaves-Castaño, L., Noreña, B. M. I., y Piñeres-Sus, J. D. (2016). Percepción del riesgo y estrategias de afrontamiento en población expuesta y no expuesta al riesgo de inundación. Revista Interamericana de Psicología, 50(3), 331-346.

Neuner, F., Schauer, E., Catani, C., Ruf, M., \& Elbert, T. (2006). Posttsunami stress: A study of posttraumatic stress disorder in children living in three severely affected regions in Sri Lanka. Journal of Traumatic Stress, 19, 339-347.

Olff, M., Langeland, W., Draiger, N., \& Gersons, B. P. R. (2007). Gender differences in posttraumatic stress disorder. Psychological Bulletin, 133(2), 183-204. https://doi.org/10.1037/0033-2909.133.2.183.

Osofsky, H. J., Osofsky, J. D., Kronenberg, M., Brennan, A., \& Hansel, T. C. (2009). Posttraumatic stress symptoms in children after Hurricane Katrina: Predicting the need for mental health services. American Journal of Orthopsychiatry, 79(2), 212-220. http://doi.org/10.1037/a0016179.

Oviedo, H., C. \& Campo-Arias, A. (2005). Aproximación al uso del coeficiente alfa de Cronbach. Revista Colombiana de Psiquiatria, 34(4), 572-580. 
Pynoos, R., Steinberg, A., \& Brymer, M. (2007). Children and disasters: Public mental health approaches. In R. Ursano, C. Fullerton, L. Weisaeth y B. Raphael (eds.). Textbook of Disaster Psychiatry (pp. 48-68). Cambridge: Cambridge University Press.

Prince, S. A. (1920). Catastrophe and social change: Based upon a sociological study of the Halifax disaster. New York: Columbia University Press.

Rincón, P., Gysling, M, Jiménez, C., Lloyd, S., Navarro, M. F., Retamal, L. Cova, F., Valdivia, M., Paz, P. S., C., Melipillán, R. y Haquin, C. (2014). Propiedades psicométricas de la Escala de síntomas de TEPT para niños (CPSS) en población chilena afectada por el terremoto y tsunami del 27-F de 2010. Terapia Psicológica, 32(1), 57-64.

Rodríguez, M. M. F., Morell, J. M., y Fresneda, J. (2015). Manual de promoción de la resiliencia infantily adolescente. Ediciones Pirámide.

Salgado, R. J. A. y Leria, D. F. J. (2018). Estrategias de afrontamiento al estrés y síntomas patológicos en universitarios ante un desastre socionatural de aluvión de barro. Acta Colombiana de Psicología, 21(1), 170-182. http://doi.org/10.14718/acp.2018.21.1.8

Shalev, A. Y. (2005). Biological responses to disasters. American Psychiatric Association. Disponible en http:// www.psych.org/disasterpsych/sl/biologicalresponses.cfm

Salotti, P. (2006). Estudio sobre estrategias de afrontamiento y bienestar psicológico en una muestra de adolescentes de Buenos Aires. Buenos Aires: Universidad de Belgrano. Disponible en http://www.ub.edu.ar/ investigaciones/tesinas/207_salotti.pdf

SSN (Servicio Sismológico Nacional). (2017). Disponible en http://www.resnom.cicese.mx/sitio/imagnes/19septiembre2017.pdf. Consultado el 6 de marzo de 2018.

Tapia, R., Sepúlveda, J., Medina-Mora, M. E., Caraveo, J. y De la Fuente, J. R. (1987). Prevalencia del síndrome de estrés postraumático en la población sobreviviente a un desastre natural. Salud Publica México, 29(5), 406-411.

Tolin, D. F., \& Foa, E. B. (2006). Sex differences in trauma and posttraumatic stress disorder: A quantitative review of 25 years of research. Psychological Bulletin, 132(6), 959-992.

Vázquez Valverde, C., Crespo López, M. y Ring, J. M. (2003). Estrategias de afrontamiento, en A. V. Bulbena., G. E. Berrios, P. P. Fernández de Larriona (Eds.), Medición Clinica en Psiquiatría y Psicología (pp. 425-435). Barcelona: Masson.

Verduzco, M. A., Gómez-Maqueo, E. L. y Durán Patiño, C. (2004). La influencia de la autoestima en la percepción del estrés y el afrontamiento en niños de edad escolar. Salud Mental, 27(4), 18-25.

Villagrán, L., Reyes, C. y Wlodarczyk, A. (2014). Afrontamiento comunal, crecimiento postraumático colectivo y bienestar social en el contexto del terremoto del 27 de febrero de 2010 en Chile. Terapia Psicológica, 32(3), 243-254.

\section{BY-NC-ND}

